\title{
SIMPLE PROOF OF CALABI-BERNSTEIN'S THEOREM ON MAXIMAL SURFACES
}

\author{
ALFONSO ROMERO \\ (Communicated by Christopher Croke) \\ Dedicated to the memory of Professor P. Bobillo
}

The following uniqueness result, called Calabi-Bernstein's Theorem on maximal surfaces, is well known ([Ca], [C-Y], [K], [E-R]).

Theorem. The only entire solutions to the maximal surface equation

$$
\operatorname{div}\left(\frac{D u}{\sqrt{1-|D u|}^{2}}\right)=0, \quad|D u|<1
$$

are affine functions.

In the references cited above, this result appears as either a particular case of some much more general theorems or stated in terms of local complex representation of the surface. However, a direct simple proof would be desirable to be easily understood for beginning researchers. The proof we present here uses only Liouville's Theorem on harmonic functions on $\mathbb{R}^{2}$. Thus, it is simple and complex function theory is not needed. This proof is inspired by [Ch]. Roughly, the key steps of our proof are: (1) On any maximal surface there exists a positive harmonic function, which is constant if and only if the surface is totally geodesic. (2) The metric of any spacelike graph is globally conformally related to a metric $g^{*}$, which is complete when the graph is entire. (3) On any maximal graph the metric $g^{*}$ is flat.

\section{Preliminaries}

Consider the Lorentz-Minkowski space $\mathbb{L}^{3}$ with its Lorentzian metric $\langle\rangle=$, $d x_{1}^{2}+d x_{2}^{2}-d x_{3}^{2}$, given in the usual coordinate system. Let $x: M \rightarrow \mathbb{L}^{3}$ be a spacelike immersion of a two-dimensional manifold $M$ in $\mathbb{L}^{3}$. Note that $M$ must be orientable. Let $N$ be a globally defined unit timelike normal vector field on $M$. Suppose that the mean curvature of $x$ vanishes; then $M$ is called a maximal surface in $\mathbb{L}^{3}$.

For each vector $a \in \mathbb{L}^{3}$ we can consider on $M$ the smooth function $\langle N, a\rangle$. Let $a^{T}$ be the vector field on $M$ induced from the tangential component of $a$ at any

Received by the editors August 11, 1994.

1991 Mathematics Subject Classification. Primary 53C42, 53C50.

Key words and phrases. Maximal surface, entire maximal graph, Lorentz-Minkowski space.

Research partially supported by DGICYT Grant No. PB91-0731. 
point of $M$, that is, $a^{T}=a+\langle N, a\rangle N$. Standard computations give the identities

$$
\begin{gathered}
\Delta\langle N, a\rangle=\operatorname{tr}\left(A^{2}\right)\langle N, a\rangle, \\
\nabla\langle N, a\rangle=-A\left(a^{T}\right),
\end{gathered}
$$

where $A$ is the Weingarten endomorphism associated to $N$, and $\Delta$ and $\nabla$ are respectively the Laplacian and the gradient relative to the induced Riemannian metric $g$ on $M$.

From (2) we obtain

$$
|\nabla\langle N, a\rangle|^{2}=(1 / 2) \operatorname{tr}\left(A^{2}\right)\left\{\langle N, a\rangle^{2}+\langle a, a\rangle\right\} .
$$

Now, choose $a \neq 0$ satisfying $\langle a, a\rangle=0$. Note that in this case $\langle N, a\rangle$ never vanishes and thus we may assume $\langle N, a\rangle>0$. From (1) and (3) we get

$$
\Delta(1 /\langle N, a\rangle)=0 .
$$

On the other hand, if we choose now $b \in \mathbb{L}^{3}$ such that $\langle b, b\rangle=-1$ and $\langle N, b\rangle>0$, then, using again (1) and (3) we obtain

$$
\Delta \log (\langle N, b\rangle+1)=(1 / 2) \operatorname{tr}\left(A^{2}\right)
$$

which means, taking into account the Gauss equation $2 K=\operatorname{tr}\left(A^{2}\right)$ for $x$, that the metric $g^{*}=(\langle N, b\rangle+1)^{2} g$ on $M$ is flat.

\section{Proof of the Theorem}

We begin by observing that on a spacelike graph in $\mathbb{L}^{3}$ of any entire function $u=u(x, y),|D u|<1$, the metric $g^{\prime}=\left(1 /\left(1-|D u|^{2}\right)\right) g$ is complete. This easily follows from the inequality $L^{\prime} \geq(1 / \sqrt{2}) L_{0}$, where $L^{\prime}$ (resp. $L_{0}$ ) denotes the length of a curve on the graph with respect to $g^{\prime}$ (resp. the metric induced from the usual Riemannian one of $\left.\mathbb{R}^{3}\right)$. Now take $b=(0,0,-1)$ so that $\langle N, b\rangle=1 / \sqrt{1-|D u|^{2}}$. Consider now the Riemannian metric $g^{*}$ introduced above. This metric is complete on a spacelike graph of any entire function. Assume the graph is maximal. On the other hand, from (5) we see that $g^{*}$ is flat. Using Cartan's Theorem we have a global isometry from the Euclidean plane $\mathbb{R}^{2}$ onto the maximal entire graph endowed with the metric $g^{*}$. The invariance of harmonic functions under conformal changes of metric and this isometry permit us to induce $1 /\langle N, a\rangle$ on a positive harmonic function on $\mathbb{R}^{2}$ which must be constant by Liouville's Theorem. Hence $\langle N, a\rangle$ is constant on the graph, which implies, using (1), that the maximal graph is totally geodesic.

\section{REMARK}

Consider as in $\S 1$, a maximal surface $M$ in $\mathbb{L}^{3}$. The metric $g^{*}$ on $M$ is also complete if the induced metric $g$ is complete. Thus, the same reasoning as in the previous section gives a proof of the well-known parametric version of this Theorem: "The only complete maximal surfaces in $\mathbb{L}^{3}$ are the spacelike planes." 


\section{REFERENCES}

[Ca] E. Calabi, Examples of Bernstein problems for some non-linear equations, Proc. Sympos. Pure Math. 15 (1970), 223-230. MR 41:8806

[Ch] S. S. Chern, Simple proofs of two theorems on minimal surfaces, Enseign. Math. 15 (1969), 53-61. MR 39:7516

[C-Y] S. T. Cheng, S. T. Yau, Maximal spacelike hypersurfaces in the Lorentz-Minkowski space, Ann. of Math. 104 (1976), 407-419. MR 55:4063

[E-R] F. J. M. Estudillo, A. Romero, Generalized maximal surfaces in Lorentz-Minkowski space $\mathbb{L}^{3}$, Math. Proc. Camb. Phil. Soc. 111 (1992), 515-524. MR 93b:53010

[K] O. Kobayashi, Maximal surfaces in the 3-dimensional Minkowski space $\mathbb{L}^{3}$, Tokyo J. Math. 6 (1983), 297-309. MR 85d:53003

Departamento de Geometria y Topologia, Facultad de Ciencias, Universidad de GranADA, 18071-GranADA

E-mail address: aromero@ugr.es 